\title{
Opinião de JOVEnS VÍtimas de TRAUMA SOBRE POLÍticas PÚBliCAS RELACIONADAS ÀS BEBIDAS ALCOÓLICAS
}

\author{
Ivonete Arnauts ${ }^{1}$ \\ Magda Lúcia Félix de Oliveira ${ }^{2}$
}

O estudo teve como objetivo verificar a opinião de jovens vítimas de trauma sobre políticas públicas de controle do uso de álcool. Estudo exploratório e transversal, com jovens na faixa etária entre 10 e 24 anos. Foram entrevistados 112 jovens, os quais foram classificados em três grupos de padrão de consumo de álcool. Os resultados apontaram que os jovens apresentaram falsa percepção de segurança referente ao consumo de álcool, apoiam programas e campanhas preventivas referentes ao consumo e têm pouca credibilidade no que refere-se ao cumprimento das políticas públicas para o álcool.

Descritores: Adolescente; Políticas Públicas; Etanol.

\footnotetext{
${ }^{1}$ RN, MSc, Secretaria Estadual de Saúde do Paraná, Cascavel, PR, Brazil.

2 PhD, Adjunct Professor, Universidade Estadual de Maringá, Maringá, PR, Brazil.
}

\section{Correspondência}

Magda Lúcia Félix de Oliveira

Universidade Estadual de Maringá

Av. Colombo, 5.790

Jd. Universitário

CEP 87.020-900, Maringá, PR, Brasil

E-mail: mlfoliveira@uem.br 


\section{YOUNG TRAUMA VICTIMS' OPINIONS OF PUBLIC POLICIES FOR ALCOHOLIC BEVERAGES}

The aim of this study was to discover the opinions young trauma victims have of public policies to control alcohol use. This was an exploratory, cross-sectional study involving young people aged between 10 and 24 years old. In total, 112 young people were interviewed and classified into three groups of alcohol consumption patterns. The results indicated that the young people interviewed had a false sense of safety towards alcohol consumption, supporting programs and campaigns to prevent consumption and have little credibility regarding compliance with public policies for alcohol.

Descriptors: Adolescent; Public Policy; Ethanol.

\section{OPINIÓN DE JÓVENES VÍCTIMAS DE TRAUMAS ACERCA DE LAS POLÍTICAS PÚBLICAS RELACIONADAS CON LAS BEBIDAS ALCOHÓLICAS}

El estudio tiene como objetivo verificar la opinión de jóvenes víctimas de traumas acerca de las políticas públicas de control del uso del alcohol. Estudio exploratorio y colateral, con jóvenes en la franja comprendida entre los 10 y los 24 años de edad. Fueron entrevistados 112 jóvenes, los cuales fueron clasificados en tres clases según el consumo de alcohol. Los resultados apuntaron a que los jóvenes tienen una falsa percepción de seguridad frente al consumo de alcohol, apoyan programas y campañas preventivas referentes al consumo y tienen poca confianza en lo referente al cumplimiento de las políticas públicas para las bebidas alcohólicas.

Descriptores: Adolescente; Políticas Públicas; Etanol.

\section{Introdução}

O consumo de álcool está associado a intercorrências indesejáveis, como crises familiares, atos violentos e internações hospitalares, sendo considerado um problema de saúde pública no Brasil. O trauma aparece como uma das consequências decorrentes do comportamento alterado pelo consumo de álcool. Um dos problemas mais importantes do consumo prejudicial de álcool é a incidência de acidentes de trânsito, envolvendo usuários desta droga ${ }^{(1-2)}$.

O uso prejudicial de drogas e violência no trânsito são fenômenos complexos e dinâmicos na vida em comunidade, permeados por questões sociais, econômicas e políticas. A bebida é parte da civilização, no entanto, é necessário civilizar seu uso $^{(3)}$. Reconhecendo os danos físicos, emocionais e materiais provocados pelo uso nocivo de álcool, são lavradas leis e estratégias preventivas.

No Brasil, a venda de drogas lícitas, como álcool e tabaco, é proibida para menores de 18 anos, por meio do Estatuto da Criança e do Adolescente - Lei 8.069/90, art. 243 ${ }^{(4)}$. A Lei 11.705/08, denominada Lei Seca, e a Lei 6.488/08, impõem penalidades mais coercivas ao condutor que dirigir sob influência de qualquer quantidade de álcool, como pagamento de multa, perda da carteira de motorista por 12 meses e detenção em flagrante. A legislação proíbe, também, a venda de bebidas em estabelecimentos comerciais instalados nas rodovias, no perímetro urbano das cidades.

O I Levantamento Nacional sobre os Padrões de Consumo de Álcool na População Brasileira, realizado em 2007, apontou que os jovens são os que apresentam maiores riscos em relação 
ao consumo de álcool, com consequências negativas diversas, como "[...] problemas nos estudos, problemas sociais, (prática de) sexo sem proteção e/ou sem aconselhamento, maior risco de suicídio ou homicídio, e acidentes [... ${ }^{\prime \prime(5)}$. Os jovens são população vulnerável, por passarem por profundas mudanças físicas e psíquicas ${ }^{(5-6)}$.

Medidas legais adotadas por governos são importantes para impedir o acesso dos adolescentes às drogas, porém, a legislação nem sempre é cumprida, predispondo à aquisição de drogas pelos jovens ${ }^{(7)}$.

A presente pesquisa justifica-se, então, pela relevância científica e social dos estudos sobre abuso de álcool e outras drogas, e pela estreita ligação entre aumento do consumo de álcool e risco de trauma, principalmente no grupo populacional dos jovens.

O estudo teve como objetivo compreender o que pensam os jovens que sofreram trauma, relacionado ou não ao uso de bebidas alcoólicas, sobre uso de álcool e políticas relacionadas.

\section{Metodologia}

O estudo foi do tipo exploratório e transversal, com 112 jovens com diagnóstico médico de trauma, por diversas etiologias, na faixa etária entre 10 e 24 anos, residentes em Cascavel - PR, atendidos no Pronto Socorro (PS) de um hospital de ensino do Oeste do Paraná. A faixa etária escolhida para o estudo está em consonância com o conceito de jovem empregado por Nugent (2006), com três fases de transição da juventude: 10 a 14 anos, 15 a 20 anos e 21 a $24 \operatorname{anos}^{(8)}$.

A identificação dos participantes ocorreu através da listagem de pacientes atendidos no PS, o prontuário do paciente e a ficha de atendimento do PS. Os instrumentos de coleta de dados foram um roteiro de entrevista, adaptado do Questionário Hablas, desenvolvido pela equipe do professor Raul Caetano, da Universidade do Texas - EUA e uma planilha para compilação de dados.

As entrevistas foram realizadas entre maio e julho de 2009, às sextas-feiras, sábados e domingos, das dezenove horas à uma hora. Optou-se por este horário para obtenção de maior número de sujeitos, tratando-se, portanto, de uma amostra não probabilística, a população não estava disponível para ser sorteada ${ }^{(9)}$.
Os dados foram inseridos no Programa Excel, para posterior análise através do Programa de Computação Estatística 8.0. A análise de dados foi realizada por meio de estatística descritiva, teste quiquadrado e regressão logística. $\mathrm{O}$ projeto foi aprovado pelo parecer n. ${ }^{\circ}$ 070/2009, do Comitê Permanente de Ética e Pesquisa Envolvendo Seres Humanos, da Universidade Estadual de Maringá.

\section{Resultados}

Foram entrevistados 112 jovens, classificados em três grupos de padrão de consumo de álcool: $22(19,7 \%)$ que tiveram trauma associado ao consumo de bebida alcoólica, denominados trauma com consumo; 65 (58\%) que eram consumidores de álcool, mas não referiram consumo nas seis horas anteriores ao trauma, designados trauma sem consumo; e $25(22,3 \%)$ que nunca consumiram bebida alcoólica, identificados como trauma em abstinentes na vida (Figura 1).

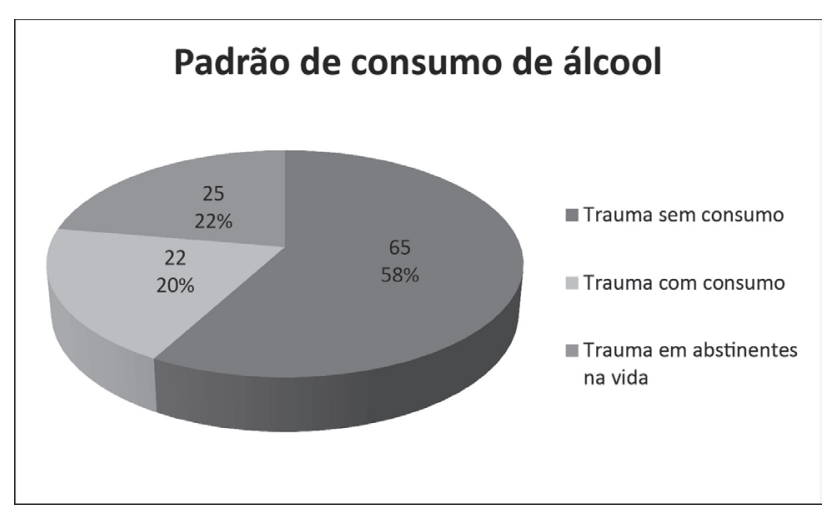

Figura 1 - Distribuição percentual de jovens vítimas de trauma, segundo padrão de consumo de álcool. Cascavel, PR, maio a julho, 2009

A caracterização socioeconômica dos jovens apontou predomínio do sexo masculino em todas as faixas etárias e em todos os grupos de padrão de consumo, sendo que a maioria dos jovens abstinentes pertenciam à faixa etária entre 10 e 14 anos. No entanto, a faixa etária predominante foi entre 15 e 20 anos, representando $42,9 \%$ de todos os jovens investigados. O grau de instrução da maioria dos jovens era o ensino fundamental completo, renda mensal familiar entre 901 e 1500 reais, sem renda pessoal e com trabalho formal. 
A opinião dos jovens vítimas de trauma sobre iniciativas e políticas públicas de controle do álcool foi avaliada por meio de questionamentos sobre legislação brasileira - impostos, comercialização, trânsito, penalidades para os crimes decorrentes do uso de bebida alcoólica - e o papel das campanhas e programas de prevenção e tratamento. Os jovens opinaram quanto ao aumento, redução e permanência dessas políticas. Para a comercialização, considerou-se aspectos favoráveis ou não favoráveis à política vigente.

Em relação aos impostos sobre as bebidas alcoólicas, entre os jovens abstinentes na vida, a maioria $(76 \%)$ considera que deveriam ser aumentados, diferente do grupo de trauma sem consumo de bebida alcoólica $(49,2 \%)$ e do grupo de trauma com consumo $(27,3 \%)$. A resposta mais citada pelos jovens que tiveram trauma relacionado ao consumo de bebida alcoólica foi de que os impostos deveriam permanecer com o mesmo valor $(45,5 \%)$. As respostas aumento e redução, para esse grupo, foram de $27,3 \%$, em ambas. Observou-se relação significativa $(p<0,05)$ quanto às opções redução, permanência ou aumento dos impostos sobre as bebidas alcoólicas, nos três grupos estudados, com resultado de Pearson Chi-square de 0,009 (Tabela 1).
Quando indagados sobre aumento, permanência ou redução da idade de 18 anos para a aquisição de bebida alcoólica, a maioria $(55,4 \%)$ optou pela permanência da idade mínima de 18 anos. No grupo dos jovens que tiveram trauma relacionado ao consumo, o percentual foi de $68,2 \%$, no grupo do trauma sem consumo, foi de $55,4 \%$, e para os abstinentes na vida foi de $44 \%$. Quanto à idade mínima para consumo de álcool, 16\% dos jovens do grupo dos abstinentes na vida apoiam a redução da idade de 18 anos para a venda de bebida alcoólica. Para $83 \%$ dos jovens entrevistados "é muito fácil para uma criança ou adolescente menor de 18 anos comprar bebida alcoólica em qualquer ponto de venda" (Tabela 1).

Nessa pesquisa, a opinião dos jovens sobre as iniciativas governamentais de controle do uso de álcool é abordada sobre os aspectos de aumento, redução e permanência das propostas atuais.

Quando questionados sobre campanhas que alertam para os riscos de ingerir bebida alcoólica e dirigir, a maioria $(86,6 \%)$ dos jovens considera que deveriam ser aumentadas, porém, entre aqueles que optaram pela diminuição, o grupo dos jovens que tiveram trauma relacionado ao consumo de bebida alcoólica apresentaram um percentual maior de respostas, quando comparados aos demais grupos $(9,1 \%)$.

Tabela 1 - Distribuição absoluta e percentual de jovens vítimas de trauma quanto à opinião sobre políticas públicas para controle do álcool. Cascavel, PR, maio a julho, 2009

\begin{tabular}{|c|c|c|c|c|c|c|c|c|c|}
\hline \multirow{2}{*}{ Opinião sobre o uso de bebida alcoólica } & \multirow{2}{*}{$\begin{array}{l}\text { Padrão de } \\
\text { consumo }\end{array}$} & \multicolumn{2}{|c|}{$\begin{array}{c}\text { Trauma sem } \\
\text { consumo }\end{array}$} & \multicolumn{2}{|c|}{$\begin{array}{c}\text { Trauma com } \\
\text { consumo }\end{array}$} & \multicolumn{2}{|c|}{$\begin{array}{l}\text { Trauma em } \\
\text { abst. na vida }\end{array}$} & \multicolumn{2}{|c|}{ Total } \\
\hline & & $\mathbf{N}$ & $\%$ & $\mathbf{N}$ & $\%$ & $\mathbf{N}$ & $\%$ & $\mathbf{N}$ & $\%$ \\
\hline \multirow[t]{3}{*}{ Impostos sobre as bebidas alcoólicas } & Aumento & 32 & 49,2 & 6 & 27,3 & 19 & 76,0 & 57 & 50,9 \\
\hline & Permanência & 27 & 41,5 & 10 & 45,5 & 2 & 8,0 & 39 & 34,8 \\
\hline & Redução & 6 & 9,3 & 6 & 27,3 & 4 & 16,0 & 16 & 14,3 \\
\hline \multirow[t]{3}{*}{ Idade de 18 anos para a venda de bebida alcoólica } & Aumento & 26 & 40,0 & 6 & 27,3 & 10 & 40 & 42 & 37,5 \\
\hline & Permanência & 36 & 55,4 & 15 & 68,2 & 11 & 44 & 62 & 55,4 \\
\hline & Redução & 3 & 4,6 & 1 & 4,5 & 4 & 16 & 8 & 7,1 \\
\hline \multirow{3}{*}{$\begin{array}{l}\text { É muito fácil para uma criança ou adolescente menor } \\
\text { de } 18 \text { anos de idade comprar bebida alcoólica }\end{array}$} & Concorda & 52 & 80,0 & 18 & 81,8 & 23 & 92,0 & 93 & 83,0 \\
\hline & Indiferente & 4 & 6,2 & 1 & 4,6 & - & - & 5 & 4,5 \\
\hline & Discorda & 9 & 13,8 & 3 & 13,6 & 2 & 8,0 & 14 & 12,5 \\
\hline \multirow[t]{3}{*}{ Campanhas alertando para os riscos de beber e dirigir } & Aumento & 56 & 86,2 & 18 & 81,8 & 23 & 92 & 97 & 86,6 \\
\hline & Permanência & 8 & 12,3 & 2 & 9,1 & 1 & 4 & 11 & 9,8 \\
\hline & Redução & 1 & 1,5 & 2 & 9,1 & 1 & 4 & 4 & 3,6 \\
\hline \multirow[t]{3}{*}{ Programas de prevenção ao uso de álcool em escolas } & Aumento & 61 & 93,8 & 18 & 81,8 & 22 & 88 & 101 & 90,2 \\
\hline & Permanência & 2 & 3,1 & _- & - & 1 & 4 & 3 & 2,7 \\
\hline & Redução & 2 & 3,1 & 4 & 18,2 & 2 & 8 & 8 & 7,1 \\
\hline \multirow[t]{3}{*}{ Programas de tratamento para alcoolismo } & Aumento & 57 & 87,7 & 19 & 86,4 & 23 & 92 & 99 & 88,4 \\
\hline & Permanência & 7 & 10,8 & 2 & 9,1 & 2 & 8 & 11 & 9,8 \\
\hline & Redução & 1 & 1,5 & 1 & 4,5 & - & - & 2 & 1,8 \\
\hline Total & & 65 & 58,1 & 22 & 19,6 & 25 & 22,3 & 112 & 100,0 \\
\hline
\end{tabular}


Quanto aos programas de prevenção do uso de álcool em escolas, foi indicado o aumento por $90,2 \%$ dos jovens. Maior percentual de jovens que tiveram trauma relacionado ao consumo de bebida alcoólica, em comparação com os demais grupos, relataram que os programas de prevenção do uso de álcool em escolas deveriam diminuir, totalizando 18,2\% das respostas. Quanto aos programas de tratamento, $90,2 \%$ dos jovens da presente pesquisa consideram que deveriam ser aumentados (Tabela 1).

Considerando, então, que o Código de Trânsito Brasileiro estabelece punições aos motoristas alcoolizados, tanto em acidentes com vítimas quanto em direção com risco à segurança de outros, foram incluídas na presente pesquisa perguntas sobre as penalidades ao motorista infrator. Perguntou-se sobre as condutas que deveriam ser tomadas em relação a uma pessoa que dirigisse após ingerir três ou mais doses de bebida alcoólica, sendo apresentadas as medidas de restrição de liberdade do condutor (detenção policial), carteira de habilitação $(\mathrm{CNH})$ suspensa e multa, e as variáveis nunca, em poucas situações, em várias situações e sempre.

Para as três opções de medidas legais, a variável sempre foi a mais citada pelos três grupos de jovens investigados, sendo de $54,5 \%$ para prisão do condutor, 53,6\% para $\mathrm{CNH}$ suspensa, e $75 \%$ para pagamento de multas (Tabela 2 ).

Os jovens que tiveram trauma relacionado ao consumo de bebida foram os que mais escolheram a variável nunca, quando comparados aos jovens que tiveram trauma sem consumo e jovens abstinentes na vida. Esta atitude era esperada, pois ao aprovarem a penalização em relação a este comportamento de risco, estariam se penalizando.

Tabela 2 - Distribuição absoluta e percentual de jovens vítimas de trauma quanto à opinião sobre penalidades para dirigir embriagado. Cascavel, PR, maio a julho, 2009

\begin{tabular}{|c|c|c|c|c|c|c|c|c|}
\hline \multirow{2}{*}{ Dirigir após beber três ou mais doses } & \multicolumn{2}{|c|}{$\begin{array}{c}\text { Trauma sem } \\
\text { consumo }\end{array}$} & \multicolumn{2}{|c|}{$\begin{array}{c}\text { Trauma com } \\
\text { consumo }\end{array}$} & \multicolumn{2}{|c|}{$\begin{array}{c}\text { Trauma em } \\
\text { abst. na vida }\end{array}$} & \multicolumn{2}{|c|}{ Total } \\
\hline & $\mathbf{N}$ & $\%$ & $\mathbf{N}$ & $\%$ & $\mathbf{N}$ & $\%$ & $\mathbf{N}$ & $\%$ \\
\hline \multicolumn{9}{|l|}{ Deveria ser presa } \\
\hline Sempre & 38 & 58,5 & 8 & 36,4 & 15 & 60,0 & 61 & 54,5 \\
\hline Em poucas situações & 16 & 24,6 & 5 & 22,7 & 6 & 24,0 & 27 & 24,1 \\
\hline Em várias situações & 8 & 12,3 & 3 & 13,6 & 4 & 16,0 & 15 & 13,4 \\
\hline Nunca & 3 & 4,6 & 6 & 27,3 & - & - & 9 & 8,0 \\
\hline \multicolumn{9}{|c|}{ Deveria ter a sua carteira de habilitação suspensa } \\
\hline Sempre & 37 & 56,9 & 8 & 36,4 & 15 & 60,0 & 60 & 53,6 \\
\hline Em poucas situações & 12 & 18,5 & 5 & 22,7 & 6 & 24,0 & 23 & 20,5 \\
\hline Em várias situações & 12 & 18,5 & 4 & 18,2 & 4 & 16,0 & 20 & 17,9 \\
\hline Nunca & 4 & 6,1 & 5 & 22,7 & - & - & 9 & 8,0 \\
\hline \multicolumn{9}{|l|}{ Deveria ter que pagar multas } \\
\hline Sempre & 48 & 73,8 & 12 & 54,5 & 24 & 96,0 & 84 & 75,0 \\
\hline Em poucas situações & 7 & 10,8 & 6 & 27,3 & 1 & 4,0 & 14 & 12,5 \\
\hline Em várias situações & 8 & 12,3 & 2 & 9,1 & - & - & 10 & 8,9 \\
\hline Nunca & 2 & 3,1 & 2 & 9,1 & - & - & 4 & 3,6 \\
\hline Total & 65 & 58,1 & 22 & 19,6 & 25 & 22,3 & 112 & 100,0 \\
\hline
\end{tabular}

\section{Discussão}

São consideradas políticas do álcool aquelas que relacionam álcool, segurança, saúde e bem-estar social. Definem-se como políticas do álcool qualquer esforço ou decisão de autoridades governamentais ou de Organizações não Governamentais (ONG), para minimizar ou prevenir problemas relacionados ao álcool ${ }^{(10)}$.
Por meio da opinião dos jovens vítimas de trauma, sobre iniciativas e políticas públicas de controle do álcool, pode-se afirmar que os jovens que tiveram trauma relacionado ao consumo de bebida alcoólica preferem que os impostos sejam reduzidos, e os abstinentes na vida preferem que sejam aumentados. Uma das justificativas para tal resultado é que o aumento dos impostos sobre bebidas alcoólicas contribui para o aumento de seu preço. 
A estratégia de aumento de preços do álcool associa-se à redução de consumo e problemas decorrentes, principalmente nos grupos adolescentes, com menor renda disponível, e dos bebedores pesados, sendo uma das formas de redução da embriaguez ao volante, em especial em jovens. Nos Estados Unidos, o aumento no preço de bebidas alcoólicas reduziu a probabilidade de dirigir embriagado em $7 \%$ dos homens e $8 \%$ das mulheres, com reduções ainda maiores entre menores de 21 anos $^{(11-12)}$.

Os jovens da pesquisa concordam sobre a aquisição legal de bebida alcoólica com idade mínima de 18 anos, corroborando com a legislação vigente, porém afirmam que a legislação não é cumprida. O artigo 81 do Estatuto da Criança e do Adolescente - ECA, Lei Federal no 8069, restringe a venda de bebida alcoólica para menores de 18 anos de idade. Caberia aos órgãos de fiscalização, sobretudo municipais, garantir a aplicação do ECA, coibindo a venda de bebida alcoólica para crianças e adolescentes ${ }^{(4)}$.

As limitações na venda de bebida alcoólica visam regular o mercado, limitando o acesso do consumidor ou regulando o contexto em que é consumida. Elevações na idade mínima para compra de bebidas, com adequada implementação e fiscalização, podem provocar reduções substanciais nos problemas relacionados ao álcool na população mais jovem, especialmente os decorrentes de acidentes de carro e violência $^{(10)}$.

O I Levantamento sobre os Padrões de Uso de Álcool na População Brasileira apontou que $95 \%$ dos entrevistados concordam sobre a facilidade da compra de bebidas alcoólicas por menores de 18 anos, porém, 95\% defendem o aumento da fiscalização dos comerciantes, em relação à venda de bebidas alcoólicas para menores ${ }^{(5)}$.

Estudos nacionais mostraram que jovens entre 13 e 17 anos conseguiram facilmente comprar bebidas alcoólicas em diferentes tipos de estabelecimento. Os jovens brasileiros convivem em contextos sociais facilitadores na venda de bebida alcoólica, que muitas vezes aceita, populariza e estimula o uso. Torna-se comum o incentivo da família nesse período de vida, pois a inserção social dos jovens passa a ser fundamental, onde situações como beber pode se tornar um meio de integração ${ }^{(10,13)}$.
A opinião dos jovens sobre as iniciativas governamentais de controle do uso de álcool, por meio da mídia, programas escolares e de tratamento, apontou que os jovens que tiveram trauma relacionado ao consumo de bebida alcoólica são menos favoráveis a tais propostas.

Um fator que prejudica a conscientização da população juvenil sobre os riscos do consumo de bebida alcoólica, além do contexto social, cultural e comunitário, são as propagandas, que associam bebida alcoólica a pessoas jovens, bonitas e momentos de intensa alegria e diversão. Apesar da descrição "esse produto é destinado a adultos", o alvo dessas propagandas são os jovens, pois os atores são belos jovens, realizando atividades compatíveis a essa faixa etária. Embora a indústria do álcool tente fazer alguma propaganda educativa como "se beber não dirija", ou "beba com moderação", seus interesses comerciais entram em conflito com medidas de saúde pública. A propaganda educativa nunca é bem produzida, nem possui os mesmos recursos e frequências nos meios de comunicação quanto a propaganda da indústria do álcool ${ }^{(10,14)}$.

A sociedade aborda as drogas ilícitas como um grande problema social, diminuindo a percepção de risco das lícitas, que também apresentam consequências graves. A legalização de apenas algumas drogas faz com que o cidadão calcule seus riscos, como se o potencial danoso fosse classificado em drogas que apresentam riscos ou não ${ }^{(13)}$.

Considerando, então, que o Código de Trânsito Brasileiro estabelece punições aos motoristas alcoolizados, tanto em acidentes com vítimas quanto em direção com risco à segurança de outros, nessa pesquisa observou-se que os jovens do grupo do trauma com consumo apresentam respostas menos favoráveis à penalização do motorista infrator.

No Brasil, os acidentes automobilísticos com vítimas, em mais da metade dos casos, apresentam concomitância com uso de álcool pelo motorista e, nos atropelamentos de pedestres, as vítimas também usaram álcool. Mudanças impostas pelas leis governamentais visam a melhoria da segurança dos veículos e da população. As autoridades reconhecem o impacto do "dirigir alcoolizado" e dispõem de instrumentos legais para prevenir e reprimir a embriaguez no trânsito ${ }^{(15)}$. 
Dirigir sob efeito de álcool ou qualquer outra substância psicoativa, segundo o artigo 165 da "Lei Seca", leva à multa, suspensão do direito de dirigir por 12 meses, retenção do veículo até apresentação de um condutor habilitado, recolhimento do documento de habilitação e detenção de seis a 36 meses. Portanto, com qualquer concentração de álcool detectada no sangue, o condutor estará sujeito a penalidades de infração $\operatorname{gravíssima}^{(16)}$.

Fato preocupante observado nessa pesquisa não foi apenas o consumo de bebida alcoólica além do limite considerado seguro, especialmente no grupo do trauma com consumo, mas o fato de acharem que são capazes de dirigir. Um fenômeno significativo é a associação entre consumo de alto risco e o comportamento de dirigir. Em $64 \%$ dos acidentes com vítimas fatais, os motoristas tinham concentração sanguínea de mais de um grama de álcool por litro $(\mathrm{g} / \mathrm{l})$ de sangue. Quando os níveis de álcool no sangue atingem valores entre 0,9 e $1,5 \mathrm{~g} / 1$, o risco de acidentes de trânsito aumenta 35 vezes, comparado com um motorista sóbrio ${ }^{(5,15)}$.

\section{Considerações finais}

A caracterização sociodemográfica dos jovens que fazem uso de álcool permitiu detectar fatores que podem favorecer a percepção de risco para o trauma, referente às políticas publicas para o álcool:

- Na opinião dos jovens que tiveram trauma com consumo, políticas de aumento de impostos sobre bebida alcoólica não deveriam ser incentivadas, demonstrando que esse grupo não tem interesse em reduzir o consumo, o que poderia reduzir os problemas decorrentes do abuso de álcool.

- A facilidade para aquisição de bebida alcoólica por adolescentes e jovens foi apontada pelos jovens de todos os grupos de consumo.

- A maioria dos jovens considera que deveriam ser aumentadas as campanhas sobre os riscos de ingerir bebida alcoólica e dirigir, os programas de prevenção do uso de álcool em escolas, e programas de tratamento; porém, entre aqueles que optaram pela diminuição, o grupo dos jovens que tiveram trauma relacionado ao consumo de bebida alcoólica apresentou percentual menor de respostas.

- A opinião dos jovens sobre as penalidades que deveriam ser tomadas quanto à direção automotiva e consumo de álcool, revelou que os jovens que tiveram trauma relacionado ao consumo de bebida foram os menos favoráveis à detenção e suspensão da CNH. Cerca da metade dos jovens acreditam na existência de penalização para o condutor alcoolizado.

Nessas condições, propostas preventivas quanto ao uso de álcool e outras drogas devem ser direcionadas à comunidade, família e juventude, visando redução do risco de morbimortalidade e resgate de valores humanos e sociais. Tais propostas devem propor intervenções que levem em consideração a multiplicidade dos sujeitos, pluralidade de relações e contexto histórico e social da sociedade onde os jovens encontram-se inseridos.

A população de jovens é responsável pela introdução de novas crenças, práticas, valores e possibilidades de mudanças na sociedade. As crenças provenientes da cultura são construídas e internacionalizadas pelas pessoas, passando a fazer parte de sua visão de mundo e influenciando na interpretação dos eventos que irão ocorrer.

Sugerem-se estudos para aprofundamento da temática e implementação de políticas e estratégias preventivas, principalmente no grupo populacional dos jovens.

\section{Referências}

1. Mesquita EM, Nunes AJ, Cohen C. Avaliação das atitudes dos estudantes de medicina frente ao abuso de drogas por colegas do meio acadêmico. Rev Psiq Clín. 2008;35:8-12.

2. Ballani TSL, Oliveira MLF. Uso de Drogas de Abuso e Evento Sentinela: construindo uma proposta para avaliação de políticas públicas. Texto Contexto-Enferm. 2007;16:488-94. 
3. Secretaria de Atenção à Saúde (DF-BR). Departamento de Ações Programáticas Estratégicas. Álcool e redução de danos: uma abordagem inovadora para países em transição. Brasília (DF): SAE; 2004. 114 p.

4. Lein ${ }^{\circ} 8.069$, de 13 de julho de 1990 (BR). Dispõe sobre o Estatuto da Criança e do Adolescente e dá outras providências. Brasília: Diário Oficial da União, 16 julho 1990. Seção 1, p. 79.

5. Laranjeira R, Pinsky I, Zaleski M, Caetano R. Secretaria I Levantamento Nacional sobre padrões de consumo de álcool na população brasileira. Brasilia (DF): Nacional Antidrogas. Departamento de Psiquiatria da Universidade Federal de São Paulo; 2007. 76 p.

6. Pratta EMM, Santos MA. Reflexões sobre as relações entre drogadição, adolescência e família: um estudo bibliográfico. Estud Psicol. 2006;3:315-22.

7. Ruiz MR, Andrade D. Family and risk factors related to alcohol consumption and smoking among children and adolescents (GuayaquilEquador). Rev. Latino-Am. Enfermagem.

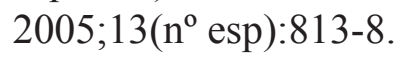

8. Nugent R. Quiénes son los jóvenes. In: Ashford L, Clifton D, Kaneda T. La juventud mundial. Washington, DC: Population Reference Bureau; 2006. p. 121-8.

9. Oliveira TM. Amostragem não probabilística: adequação de situações para uso e limitações de amostras por conveniência, julgamento e quotas. Admin Online. [Internet]. 2001 [acesso 12 fev 2011]; Disponível em: http://www.fecap.br/ adm_online/art23/tania2.htm.

10. Duailibi S, Laranjeira R. Políticas públicas relacionadas às bebidas alcoólicas. Rev Saúde Pública. 2007;41:839-48.

11. Birckmayer JD, Holder HD, Yacoubian GS $\mathrm{Jr}$, Friend KB. A general causal model to guide alcohol, tobacco, and illicit drug prevention: assessing the research evidence. J Drug Educ. 2004;34:121-53.

12. Jones-Webb R, Toomey T, Miner K, Wagenaar AC, Wolfson M, Poon R. Why and in what context adolescents obtain alcohol from adults: a pilot study. Subst Use Misuse. 1997;32:219-28.

13. Martins RA. Uso de álcool, intervenção breve e julgamento sócio-moral em adolescentes que bebem excessivamente. [Tese de LivreDocência em Psicologia da Educação]. Instituto de Biociências, Letras e Ciências Exatas da Universidade de São Paulo; 2006. 211 p.

14. Dallo L. Padrão de uso de álcool e outras drogas entre estudantes de Cascavel-PR. 2009. [Dissertação de Mestrado em Educação]. Marília: Universidade Estadual Paulista Júlio de Mesquita Filho; 2009. 106 p.

15. Farias GM, Rocha KMM, Freitas MCS, Costa IKF, Dantas RAN. Acidentes de trânsito decorrentes da influência do uso do álcool. Inter Science Place. [Internet]. 2009 [acesso 20 jan 2010];2. Disponível em: http://www.interscienceplace.org/interscienceplace/article/ view/100/102

16. Ministério da Saúde (BR). Lei no 11.705 , de 19 jun 2008. Inibe o consumo de bebida alcoólica por condutor de veículo automotor, e dá outras providências. Diário Oficial da União, Brasília, DF, 20 junho de 2008. 4 p. 